\title{
Comparison of mobilization with supervised exercise for patients with subacromial impingement syndrome
}

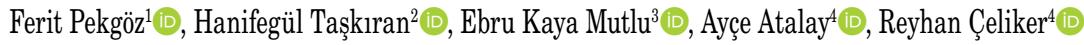 \\ 'Division of Physical Therapy and Rehabilitation, Istanbul Medipol University, Institute of Health Sciences, Istanbul, Turkey \\ ${ }^{2}$ Division of Physical Therapy and Rehabilitation, Istanbul Aydın University, Faculty of Health Sciences, Istanbul, Turkey \\ ${ }^{3}$ Division of Physical Therapy and Rehabilitation, Istanbul University, Faculty of Health Sciences, Istanbul, Turkey \\ ${ }^{4}$ Department of Physical Medicine and Rehabilitation, Acrbadem University, Faculty of Medicine, Istanbul, Turkey
}

Received: September 28, 2018 Accepted: April 10, 2019 Published online: May 18, 2020

\begin{abstract}
Objectives: This study aims to investigate the effects of joint mobilization with supervised exercise in patients with subacromial impingement syndrome (SAIS).

Patients and methods: This prospective, randomized-controlled study included a total of 40 patients (18 males, 22 females; mean age 43.52 years; range, 27 to 67 years) with SAIS of more than six weeks between June 2014 and June 2015 . The patients were randomly allocated into two groups: Group $1(n=20)$ received joint mobilization and neuromuscular electrical stimulation and Group 2 $(n=20)$ received a supervised exercise program and neuromuscular electrical stimulation. The outcome measures included the range of motion, pain intensity, the Disabilities of the Arm, Shoulder and Hand (DASH) questionnaire, American Shoulder and Elbow Surgeons Standardized Shoulder Assessment Form-patient self-report section, Short Form-36, and Global Rating of Change Questionnaire. Pain was evaluated using the visual analog scale (VAS).

Results: In both groups, the mean VAS scores significantly decreased and the range of motion significantly increased after treatment $(\mathrm{p}<0.05)$. Both joint mobilization and supervised exercise combined with neuromuscular electrical stimulation led to a significant improvement in function in patients with SAIS $(\mathrm{p}<0.05)$, although it did not significantly differ between the groups $(\mathrm{p}>0.05)$. Patient satisfaction with treatment was similar in both groups $(\mathrm{p}=0.28)$.
\end{abstract}

Conclusion: Based on our study results, mobilization and supervised exercise yield comparable outcomes in patients with SAIS.

Keywords: Exercise, joint mobilization, shoulder pain, subacromial impingement syndrome.

Subacromial impingement syndrome (SAIS) is one of the most common entities encountered in the shoulder complex characterized by pain and functional restrictions. This condition is due to the impingement of the tendons of the rotator cuff, particularly the supraspinatus tendon, head of the biceps brachii, and subacromial bursa between the head of the humerus and coracoacromial arch. The main goals of SAIS treatment are to control pain and increase the range of motion (ROM) in order to enable healing of the compromised rotator cuff. Various programs including electrotherapy, manual therapy, passive, active and active assistive ROM exercises, and stretching and strengthening exercises to the rotator cuff and thoracoscapular muscles are frequently used for the conservative treatment of this complex process..$^{[1-3]}$

Exercise therapy is associated with successful outcomes comparable to surgery. In a recent systematic review and meta-analysis of randomized-controlled trials regarding the effectiveness of conservative measures with shoulder impingement, manual therapy plus exercise was found to be superior to exercise alone. ${ }^{[1]}$ Several studies have demonstrated the beneficial effects of exercise in patients with SAIS, particularly in terms of pain and function. ${ }^{[2]}$ However, manual therapies seem to be controversial compared to exercise, since evidence is low-to-moderate in 
case of SAIS. ${ }^{[3]}$ In the most previous research, manual therapy was investigated as an add-on treatment modality. In our study, we aimed to compare manual therapy versus exercise in patients with SAIS of which both were combined with neuromuscular electrical stimulation (NMES), which is widely used as an adjunct to physical therapy programs in daily practice.

\section{PATIENTS AND METHODS}

This parallel-design, prospective, randomizedclinical study was conducted at Maslak Acrbadem Hospital, Physical Therapy and Rehabilitation outpatient clinic between June 2014 and June 2015. Of a total of 52 patients with SAIS, 49 who fulfilled the inclusion criteria were included. Inclusion criteria were as follows: age $\geq 18$; pain lasting for $\geq 6$ weeks; having three of the following five clinical signs of SAIS (i) positive Hawkins test, (ii) positive Neer test, (iii) pain during active arm elevation of greater than $60^{\circ}$ in the scapular or sagittal plane, (iv) positive Jobe/empty can test for pain or weakness, and $(v)$ pain or weakness with resisted shoulder external rotation with the arm at the side. ${ }^{[4]}$ Patients were excluded if they had a history of shoulder or cervical or thoracic spine surgery, having another physiotherapy treatment of this disorder within the past six months, steroid injection around the shoulder within the past six months, contraindications to manual therapy such as systemic arthritis or osteoporosis, and a history of psychiatric treatment. Of 49 patients, three discontinued after the pre-evaluation phase and 23 were randomized to two groups. However, six patients discontinued treatment; therefore, a total of 40 patients (18 males, 22 females; mean age 43.52 years; range, 27 to 67 years) were included in the study. The study flow diagram is shown in Figure 1. A written informed consent was obtained from each patient. The study protocol was approved by the Istanbul Medipol University, Faculty of Medicine, Human Research Ethics Committee (No: 29, Date: 03/03/2014). The study was conducted in accordance with the principles of the Declaration of Helsinki. All patients were randomly assigned to one of two parallel groups to receive either joint mobilization combined with NMES (Group 1, $\mathrm{n}=20$ ) or exercise combined with NMES (Group 2, $n=20$ ) at a ratio of 1:1. To allocate the participants, the Research Randomizer, which is an online randomization web service (https://www. randomizer.org/) was used. Simple randomization procedures (computerized random numbers) were conducted and sequentially numbered index cards with the random assignment were prepared by an investigator with no clinical involvement in the study.
The index cards were folded and placed in sealed, opaque envelopes. Then, the blind investigator opened each envelope and allocated the participants to Group 1 or Group 2 according to the selected index card. The interventions were performed by a single physical therapist at a research clinic of a tertiary hospital, and the assessments and data collection were conducted by another therapist. The interventionist was unblinded to the allocated arm, while the patients and the outcome assessor were blinded to the allocation procedure. All participants received treatment at the clinic (three times a week) for 12 sessions for $50 \mathrm{~min}$. Outcomes were measured at baseline and at the end of treatment.

\section{Treatment protocol}

The patients were seated with their shoulder in $45^{\circ}$ of abduction, neutral rotation, and $15^{\circ}$ of horizontal adduction. Their hips and knees were flexed to $90^{\circ}$ and their feet resting flat on the floor. The NMES was applied to the lower and middle trapezius muscles. Pad placement was assisted by palpation and visual localization of the muscle during a resisted isometric contraction. The portable NMES device was used which stimulates efferent motor neurons with biphasic waveform at a frequency of 50 pulses per second, pulse length of 300 microsec, and one-sec ramp time. The electrical stimulation amplitude was applied according to the maximal perceived tolerance of the patient.

Passive joint mobilization includes shoulder distraction, dorsal glide, ventral glide, lateral glide, and caudal glide. In shoulder distraction, dorsal glide and caudal glide were applied by the physical therapist, when the patient was in supine position with shoulder $50^{\circ}$ abduction and $30^{\circ}$ horizontal adduction. The lateral glide was applied, when the patient was in the supine position with shoulder $90^{\circ}$ flexion.

Exercises were carried out under the supervision of the physiotherapist for three times a week, for a total of 12 sessions. During each session, the patients were observed for substitution or compensatory movements and corrected when required. We aimed to increase the ROM through Codman and wand exercises in the early phase. ${ }^{[5]}$ Exercises used in this phase includes posterior capsular stretches. Once ROM is normalized, the second phase starts. Strengthening includes internal and external rotations (i.e., infraspinatus, teres minor, and subscapularis) using TheraBand, followed by abduction and forward flexion. ${ }^{[6]}$ These exercises are carried out in a short arc of 30 to 45 degrees. Female patients used yellow, while male patients used red TheraBand for strengthening exercises. Scapular stabilization exercises and deltoid 


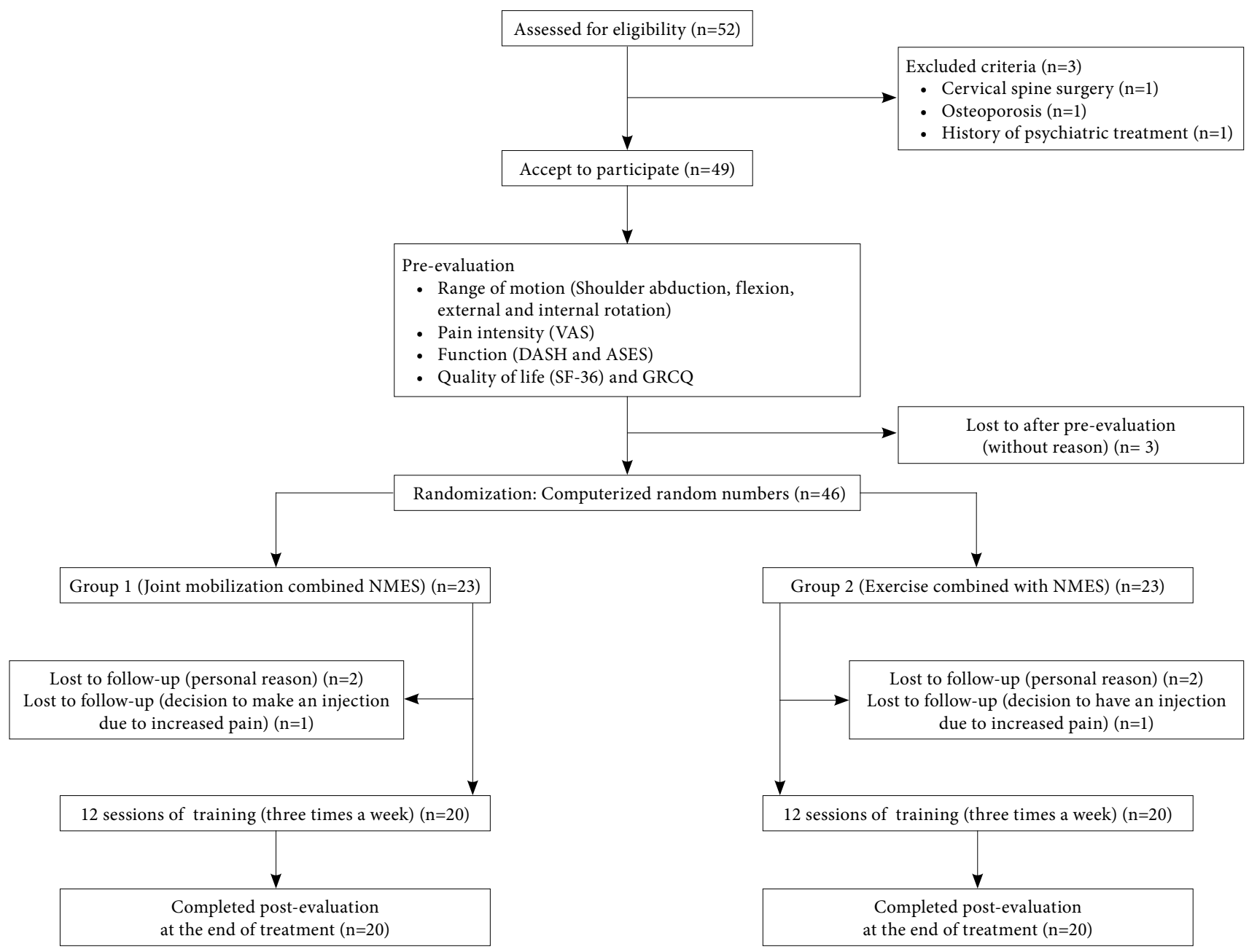

Figure 1. CONSORT flow diagram.

VAS: Visual analog scale; DASH: Disabilities of the Arm, Shoulder and Hand; ASES: American Shoulder and Elbow Surgeons; SF-36: Short Form-36; NMES: Neuromuscular electrical stimulation; GRCQ: Global Ratings of Change Questionnaire.

exercises are included in the second phase of the rehabilitation program. Exercises according to weeks are summarized in Table 1.

\section{Outcome measurements}

The outcome measures included ROM measurements, pain intensity, the Disabilities of the Arm, Shoulder and Hand (DASH) Questionnaire, American Shoulder and Elbow Surgeons (ASES) Standardized Shoulder Assessment Form-patient selfreport section, Short Form-36 (SF-36), and Global Rating of Change Questionnaire (GRCQ).

The passive ROM of each subject included abduction in the frontal plane, forward flexion, and external and internal rotation in $30^{\circ}$ abduction with a conventional goniometry. The passive ROM measurements were taken in the supine position and with the scapula being stabilized by the tester's hand. The patient's arm was passively positioned in $30^{\circ}$ abduction, as measured by a conventional goniometry, to assess internal and external rotation ROM.

The pain severity was assessed using the visual analog scale (VAS), for which a patient was asked to indicate his/her perceived pain. It was evaluated during three separate occasions: VAS on motion, VAS at night, and VAS at rest. Pain intensity was measured from the left, with 0 indicating no pain and 10 indicating severe pain. Prior to the beginning of the study and at the end of the treatment (Week 6), the VAS was administered by a physical therapist who was blind to the patient's group or treatment.

The DASH questionnaire is a reliable tool for the evaluation of functional disability of the upper 
extremity. It includes 30 questions which measure the degree of difficulty experienced due to an arm, shoulder, or hand problem (21 items) while performing various physical activities. The severity of each symptom is assessed in terms of five aspects: pain, activity-related pain, tingling, weakness and stiffness. The patient is asked to rate the impact of the problem on his social activities, work and sleep, as well as its psychological impact (4 items). Response choices for each item range from 1 to 5 , which are defined as 'no difficulty or symptom' to 'unable to perform activity or very severe symptom'.

\section{TABLE 1}

Exercise program details

Week $1\left(1^{\text {st }}, 2^{\text {nd }}\right.$ and $3^{\text {rd }}$ sessions $)$

1. Wand exercise. Shoulder abduction in the scapular plane (15 reps)

2. Wand exercise. Shoulder flexion (15 reps)

3. Wand exercise. Shoulder external rotation (towel under armpit) (15 reps)

4. Wand exercise. Shoulder extension (15 reps)

5. Wand exercise. Shoulder internal rotation (15 reps)

6. Crossed arm stretch (posterior capsular stretch) (15 reps)

Week $2\left(4^{\text {th }}, 5^{\text {th }}\right.$ and $6^{\text {th }}$ sessions $)$

1. Wand exercise. Shoulder abduction in the scapular plane (20 reps)

2. Wand exercise. Shoulder flexion (20 reps)

3. Wand exercise. Shoulder external rotation (towel under armpit) (20 reps)

4. Wand exercise. Shoulder extension (20 reps)

5. Wand exercise. Shoulder internal rotation (20 reps)

6. Crossed arm stretch (posterior capsular stretch) (20 reps)

7. External rotation in side lying (10 reps)

8. Scaption (10 reps)

9. Serratus anterior (supine) with $1 \mathrm{~kg}$ dumbbell (10 reps)

10. Scapular retraction (10 reps)

Week $3\left(7^{\text {th }}, 8^{\text {th }}\right.$ and $9^{\text {th }}$ sessions $)$

1. Wand exercise. Shoulder abduction in the scapular plane (25 reps)

2. Wand exercise. Shoulder flexion (25 reps)

3. Wand exercise. Shoulder external rotation (towel under armpit) (25 reps)

4. Wand exercise. Shoulder extension (25 reps)

5. Wand exercise. Shoulder internal rotation (25 reps)

6. Crossed arm stretch (posterior capsular stretch) (25 reps)

7. External rotation in side lying with $1 \mathrm{~kg}$ dumbbell (15 reps)

8. Scaption with $1 \mathrm{~kg}$ dumbbell (15 reps)

9. Serratus anterior (supine) with $1 \mathrm{~kg}$ dumbbell (15 reps)

10. Scapular retraction (prone) with $1 \mathrm{~kg}$ dumbbell (15 reps)

Week $4\left(10^{\text {th }}, 11^{\text {th }}, 12^{\text {th }}\right.$ sessions $)$

1. Crossed arm stretch (posterior capsular stretch) (20 reps)

2. External rotation in side lying with $1.5 \mathrm{~kg}$ dumbbell (20 reps)

3. Scaption with $1.5 \mathrm{~kg}$ dumbbell (20 reps)

4. Serratus anterior (supine) with $1.5 \mathrm{~kg}$ dumbbell (20 reps)

5. Scapular retraction (prone) $1.5 \mathrm{~kg}$ dumbbell (20 reps)

6. External rotation during standing with TheraBand (20 reps)

7. Scaption with TheraBand (20 reps) 
The scores obtained from all items are, then, used to calculate a score ranging from 0 (no disability) to 100 (most severe disability). The DASH has an excellent reliability (intra-class coefficient $=0.92,0.96$ ) and responsiveness. ${ }^{[7]}$ The validity and reliability of the Turkish version of DASH have been shown previously. ${ }^{[8]}$ Although success is defined as $50 \%$ improvement in DASH, $40 \%$ change from baseline is also accepted as a substantial improvement. ${ }^{[9,10]}$

The patient self-reported section of the ASES Standardized Shoulder Assessment form was used to evaluate patient-rated shoulder pain and function. The minimal clinically important difference (MCID) is 6.4 ASES points. ${ }^{[1]}$ The Turkish ASES questionnaire has been shown to be valid and reliable previously. ${ }^{[12]}$ The quality of life was measured using the SF-36. Higher scores indicate a better health state. ${ }^{[13]}$

The GRCQ is a six-point scale, ranging from -2 (a very great deal worse) to 0 (about the same) to +2 (a very great deal better), allowing the patient to rate the perceived change in their shoulder condition since his/her first visit. Success was defined as GRCQ scored as "moderately better" $(+3)$ or higher. ${ }^{[9]}$

\section{Statistical analysis}

The sample size and study power were calculated using the $G^{*}$ Power version 3.1.9.7 software (HeinrichHeine-Universität Düsseldorf, Düsseldorf, Germany). The calculations were based on a standard deviation of 20 points, and an inter-group difference of 15 points $^{[5]}$ represented the MCID DASH at an alpha level of 0.05 , and beta level of $20 \%$ at a desired power of $80 \%$. Accordingly, a sample size of at least 21 patients in each group was estimated. Allowing for a conservative dropout rate, we recruited 26 subjects in the study.
Statistical analysis was performed using the SPSS version 16.0 software (SPSS Inc., Chicago, IL, USA). Descriptive data were expressed in mean \pm standard deviation (SD), median (min-max) or number and frequency. Continuous variables were assessed using the independent samples t-test, while categorical variables were assessed using the chi-square test. The ROM, VAS, DASH, ASES, and SF-36 scores were analyzed using a two-by-two repeated-measures analysis of variance (ANOVA) with the treatment group (Groups 1 and 2) as the between-subject factor and time (before and after six weeks) as the within-subject factor. Pre- and post-treatment values within the groups were compared using the paired samples t-test. A $p$ value of $<0.05$ was considered statistically significant.

\section{RESULTS}

Baseline demographic characteristics of the study group are summarized in Table 2. Both groups had similar characteristics in terms of all variables, except for body mass index (BMI). Group 1 had significantly higher BMI values compared to Group $2(\mathrm{p}=0.01)$.

After the treatment, both groups had similar improvements in terms of ROM (Table 3). Both groups showed statistically significant improvements after treatment $(\mathrm{p}<0.05)$, indicating no statistically significant difference between the groups $(\mathrm{p}>0.05)$. The VAS, DASH, and ASES scores were also significantly improved after the treatment within each group $(\mathrm{p}<0.05)$ (Table 4). However, the VAS, DASH, and ASES scores did not significantly differ between the groups ( $\mathrm{p}>0.05$ ) (Table 4).

In both groups, treatment yielded a significantly improved physical and social function domain of SF-36, but not role-physical, pain, vitality, and

\begin{tabular}{|c|c|c|c|c|c|}
\hline \multicolumn{6}{|c|}{$\begin{array}{c}\text { TABLE } 2 \\
\text { Baseline demographic characteristics of study groups }\end{array}$} \\
\hline & \multicolumn{2}{|c|}{ Group $1(n=20)$} & \multicolumn{2}{|c|}{ Group $2(n=20)$} & \multirow[b]{2}{*}{$p$} \\
\hline & $\mathrm{n}$ & Mean \pm SD & $\mathrm{n}$ & Mean \pm SD & \\
\hline Age (years) & & $45.2 \pm 6.5$ & & $41.9 \pm 90$ & $0.19^{*}$ \\
\hline Body mass index $\left(\mathrm{kg} / \mathrm{m}^{2}\right)$ & & $27.1 \pm 4.5$ & & $24.1 \pm 2.9$ & $0.01^{\star}$ \\
\hline Gender & & & & & $0.05^{\star *}$ \\
\hline Female & 8 & & 14 & & \\
\hline Male & 12 & & 6 & & \\
\hline Handedness & & & & & $0.51^{\star *}$ \\
\hline Involved dominant & 11 & & 13 & & \\
\hline Non-dominant & 9 & & 7 & & \\
\hline
\end{tabular}




\begin{tabular}{|c|c|c|c|c|c|}
\hline \multirow[b]{3}{*}{ Assessments } & a- and inter-grou & $\begin{array}{c}\text { TABLE } 3 \\
\text { comparison of }\end{array}$ & assive $r$ & nge of motion & \multirow[b]{3}{*}{$p^{\star *}$} \\
\hline & Before treatment & After treatment & \multirow[b]{2}{*}{$p^{*}$} & \multirow[t]{2}{*}{ With-in score change } & \\
\hline & Mean \pm SD & Mean \pm SD & & & \\
\hline \multicolumn{6}{|l|}{ Flexion } \\
\hline Group 1 & $158 \pm 10.4$ & $170.8 \pm 10.3$ & $<0.001$ & $12.8 \pm 8.7$ & \multirow{2}{*}{0.09} \\
\hline Group 2 & $152.6 \pm 10.3$ & $166 \pm 6.8$ & $<0.001$ & $14.3 \pm 6.6$ & \\
\hline \multicolumn{6}{|l|}{ Extension } \\
\hline Group 1 & $52.5 \pm 8.6$ & $57.1 \pm 9.9$ & 0.001 & $4.6 \pm 4.0$ & \multirow{2}{*}{0.30} \\
\hline Group 2 & $46.1 \pm 5.7$ & $52.4 \pm 6.7$ & 0.001 & $6.3 \pm 6.4$ & \\
\hline \multicolumn{6}{|l|}{ Abduction } \\
\hline Group 1 & $140.7 \pm 30.0$ & $166.8 \pm 12.2$ & $<0.001$ & $26.1 \pm 29.6$ & \multirow{2}{*}{0.84} \\
\hline Group 2 & $144.4 \pm 13.9$ & $161.1 \pm 13.6$ & $<0.001$ & $16.7 \pm 9.0$ & \\
\hline \multicolumn{6}{|c|}{ Internal rotation } \\
\hline Group 1 & $54.1 \pm 6.8$ & $67.9 \pm 2.9$ & $<0.001$ & $13.8 \pm 6.0$ & \multirow{2}{*}{0.26} \\
\hline Group 2 & $52.0 \pm 4.2$ & $67.2 \pm 4.4$ & $<0.001$ & $15.2 \pm 4.7$ & \\
\hline \multicolumn{6}{|c|}{ External rotation } \\
\hline Group 1 & $71.1 \pm 4.2$ & $76.3 \pm 6.0$ & 0.001 & $5.2 \pm 5.0$ & \multirow{2}{*}{0.38} \\
\hline Group 2 & $69.1 \pm 10.4$ & $75.0 \pm 5.9$ & 0.008 & $5.9 \pm 8.9$ & \\
\hline
\end{tabular}

TABLE 4

\begin{tabular}{|c|c|c|c|c|c|}
\hline \multirow[b]{3}{*}{ Assessments } & ntra- and inter-gr & $\begin{array}{c}\text { TABLE } 4 \\
\text { up comparison }\end{array}$ & f pain a & dunction & \multirow[b]{3}{*}{$p^{* *}$} \\
\hline & Before treatment & After treatment & \multirow[b]{2}{*}{$p^{*}$} & \multirow[t]{2}{*}{ With-in score change } & \\
\hline & Mean \pm SD & Mean \pm SD & & & \\
\hline \multicolumn{6}{|l|}{ VAS-motion } \\
\hline Group 1 & $6.7 \pm 1.7$ & $3.3 \pm 1.1$ & $<0.001$ & $3.4 \pm 1.4$ & \multirow{2}{*}{0.92} \\
\hline Group 2 & $7.5 \pm 0.9$ & $4.1 \pm 1.3$ & $<0.001$ & $3.4 \pm 1.8$ & \\
\hline \multicolumn{6}{|l|}{ VAS-night } \\
\hline Group 1 & $6.1 \pm 2.2$ & $3.0 \pm 1.2$ & $<0.001$ & $3.1 \pm 1.6$ & \multirow{2}{*}{0.18} \\
\hline Group 2 & $7.3 \pm 1.6$ & $3.1 \pm 2.2$ & $<0.001$ & $4.2 \pm 1.8$ & \\
\hline \multicolumn{6}{|l|}{ VAS-rest } \\
\hline Group 1 & $3.7 \pm 1.6$ & $1.5 \pm 0.8$ & 0.001 & $2.2 \pm 1.8$ & \multirow{2}{*}{0.20} \\
\hline Group 2 & $4.6 \pm 1.8$ & $1.5 \pm 1.3$ & $<0.001$ & $3.1 \pm 1.8$ & \\
\hline \multicolumn{6}{|l|}{ DASH } \\
\hline Group 1 & $38.9 \pm 16.5$ & $22.8 \pm 11.0$ & 0.001 & $16.2 \pm 12.6$ & \multirow{2}{*}{0.18} \\
\hline Group 2 & $42.2 \pm 19.4$ & $31.9 \pm 15.3$ & 0.004 & $10.3 \pm 13.9$ & \\
\hline \multicolumn{6}{|l|}{ ASES } \\
\hline Group 1 & $59.4 \pm 7.8$ & $55.1 \pm 5.5$ & 0.008 & $8.4 \pm 7.8$ & \multirow{2}{*}{0.26} \\
\hline Group 2 & $64.2 \pm 10.7$ & $56.8 \pm 5.3$ & 0.001 & $11.1 \pm 10.6$ & \\
\hline
\end{tabular}




\begin{tabular}{|c|c|c|c|c|c|}
\hline \multirow[b]{3}{*}{ Assessments } & nd inter-group co & $\begin{array}{c}\text { TABLE } 5 \\
\text { mparison of Sho }\end{array}$ & Form- & 6 subscale scores & \multirow[b]{3}{*}{$p^{* *}$} \\
\hline & Before treatment & After treatment & \multirow[b]{2}{*}{$p^{*}$} & \multirow[t]{2}{*}{ With-in score change } & \\
\hline & Mean \pm SD & Mean \pm SD & & & \\
\hline \multicolumn{6}{|c|}{ Physical function } \\
\hline Group 1 & $73.5 \pm 15.5$ & $81.0 \pm 10.5$ & 0.04 & $7.5 \pm 15.3$ & \multirow{2}{*}{0.73} \\
\hline Group 2 & $69.8 \pm 14.0$ & $82.5 \pm 8.4$ & 0.001 & $12.8 \pm 14.2$ & \\
\hline \multicolumn{6}{|l|}{ Role-physical } \\
\hline Group 1 & $52.5 \pm 47.2$ & $57.5 \pm 46.0$ & 0.33 & $5.0 \pm 22.4$ & \multirow{2}{*}{0.68} \\
\hline Group 2 & $46.3 \pm 45.4$ & $52.5 \pm 45.8$ & 0.52 & $6.3 \pm 42.8$ & \\
\hline \multicolumn{6}{|l|}{ Pain } \\
\hline Group 1 & $43.6 \pm 19.9$ & $48.3 \pm 14.7$ & 0.31 & $4.7 \pm 20.2$ & \multirow{2}{*}{0.36} \\
\hline Group 2 & $40.7 \pm 16.6$ & $43.1 \pm 21.6$ & 0.92 & $2.4 \pm 27.0$ & \\
\hline \multicolumn{6}{|c|}{ General health } \\
\hline Group 1 & $73.0 \pm 12.2$ & $71.0 \pm 13.5$ & 0.11 & $-2.0 \pm 5.2$ & \multirow{2}{*}{0.08} \\
\hline Group 2 & $62.5 \pm 15.7$ & $63.9 \pm 12.5$ & 0.35 & $1.4 \pm 6.3$ & \\
\hline \multicolumn{6}{|l|}{ Vitality } \\
\hline Group 1 & $72.3 \pm 12.2$ & $72.0 \pm 11.4$ & 0.85 & $-0.3 \pm 7.5$ & \multirow{2}{*}{0.20} \\
\hline Group 2 & $58.5 \pm 15.1$ & $57.5 \pm 15.0$ & 0.31 & $-1.0 \pm 4.5$ & \\
\hline \multicolumn{6}{|c|}{ Social function } \\
\hline Group 1 & $77.5 \pm 22.8$ & $66.3 \pm 21.9$ & 0.03 & $-11.3 \pm 22.2$ & \multirow{2}{*}{0.17} \\
\hline Group 2 & $70.0 \pm 23.8$ & $50.0 \pm 26.9$ & 0.001 & $-20.0 \pm 20.8$ & \\
\hline \multicolumn{6}{|c|}{ Role-emotional } \\
\hline Group 1 & $78.3 \pm 36.3$ & $78.3 \pm 37.9$ & 0.99 & $-0.0 \pm 15.3$ & \multirow{2}{*}{0.30} \\
\hline Group 2 & $60.0 \pm 42.7$ & $63.2 \pm 40.4$ & 0.57 & $3.2 \pm 18.4$ & \\
\hline \multicolumn{6}{|l|}{ Mental health } \\
\hline Group 1 & $77.6 \pm 8.4$ & $72.8 \pm 7.2$ & 0.001 & $-4.8 \pm 5.1$ & \multirow{2}{*}{0.90} \\
\hline Group 2 & $74.8 \pm 7.1$ & $75.0 \pm 7.1$ & 0.66 & $0.2 \pm 2.0$ & \\
\hline
\end{tabular}

role-emotional domains. The SF-36 scores before and after the treatment are summarized in Table 5. The SF-36 subscale scores did not significantly differ between the groups ( $>0.05)$ (Table 5).

Patient satisfaction as assessed by the GRCQ was similar in both groups $(4.3 \pm 0.6$ in Group 1 vs. $4.4 \pm 0.8$ in Group 2; $\mathrm{p}=0.28$ ).

\section{DISCUSSION}

In this study, we demonstrated that, both mobilization and supervised exercise combined with NMES led to a significant improvement in function, pain, and ROM in patients with SAIS, as evidenced by the DASH and ASES scores.

Previous studies have compared manual therapy techniques with therapeutic exercise. Bang and
Deyle $^{[14]}$ compared joint mobilization as an add-on therapy to exercise (flexibility and strength training) and found that manual physical therapy combined with supervised exercise was better than exercise alone for increasing the strength, decreasing pain, and improving function in patients with SAIS. On the other hand, Camargo et al. ${ }^{[15]}$ showed that adding scapular manual therapy to an exercise protocol did not enhance improvements in scapular kinematics, function, and pain in individuals with SIAS. Kachingwe et al. ${ }^{[16]}$ also examined the effects of glenohumeral mobilizations and mobilizationwith-movement (MWM) combined with supervised exercise. They also included supervised exercise alone group and control group. The MWM and mobilization groups had a higher percentage of change in terms of pain and three interventions groups showed a better functional gain, compared to control group; however, 
these findings were not statistically significant. Also, in the literature, the MWM interventions examined in later studies were found to be more effective than placebo. ${ }^{[17,18]}$ In our study, the same effectiveness of joint mobilization for patients with SIAS was observed compared to exercise therapy. Of note, there are certain limitations to the use of manual therapy. There is poor accuracy and reproducibility of manual accessory movement testing. ${ }^{[19]}$ Thus, it is not possible to grade mobility as a dependent in studies using manual techniques. In addition, it is not possible to include a placebo group due to the ethical reasons. ${ }^{[20]}$

In clinical practice, SIAS is usually treated with stretching and strengthening exercises; however, there is no consensus which type of exercise is the most effective method. ${ }^{[3]}$ Some authors suggested that a specific exercise strategy with special focus on correction of kinematic deficits would be superior to general exercise strategy, while some others did not. ${ }^{[21]}$ On the other hand, a systemic review found that nonsteroidal anti-inflammatory drugs and corticosteroids were superior to placebo, although it was unclear how these treatments were compared to exercise. ${ }^{[1]}$ Therefore, in our study, strengthening and stretching exercises were used. Our study results indicated that either strengthening and stretching exercise or manual therapy combined with NMES yielded benefits on pain, ROM, and function.

The NMES is a widely used intervention during rehabilitation to increase the isometric strength of the muscle, and improve function and motor control, facilitating changes in muscle action and performance. To stimulate the lower trapezius and serratus anterior muscles in healthy participants, the effect of these muscles on acromiohumeral distance using NMES was previously investigated. ${ }^{[22]}$ The authors found that acromiohumeral distance increased during contraction via NMES of the lower trapezius, serratus anterior, and the combination of the two muscles. In addition, the restoration of parascapular muscles for scapular control was recommended as the strategy to restore the shoulder function with exercise. Therefore, in a conservative approach, correcting neuromuscular control of the scapular muscles is crucial. In the light of these data, our findings suggested that NMES might be an important factor for the treatment of SIAS.

Currently, there is a vast number of instruments for measuring function of the shoulder and upper extremity. The MCID of an outcome measure is the minimum change in a score which indicates a change in disability. ${ }^{[23]}$ Our study highlights the importance of a clinically important difference, as DASH and ASES scores statistically significantly improved. We used the self-report section of ASES similar to Mulligan et. ${ }^{[20]}$ In their study, axioscapular and rotator cuff exercises led to a significant improvement and the authors used the ASES as an outcome measure. Similarly, in our patients, the mean ASES scores decreased in the both groups with a clinically significant decline in the mobilization group.

The main limitations of our study include that the results were limited to follow-up outcomes of 12 sessions of training only and our sample size was diminished due to a dropout rate of $4.76 \%$. However, the main strength of our study includes its prospective, randomized-controlled design.

In conclusion, both mobilization and supervised exercises combined with NMES are effective for the treatment of patients with SAIS. In this study, we were able to compare both therapies from various aspects as the sole interventions, not as an add-on treatment modality. However, further large-scale, prospective, randomized studies are needed to confirm whether these trends in improvement are dependable.

\section{Declaration of conflicting interests}

The authors declared no conflicts of interest with respect to the authorship and/or publication of this article.

\section{Funding}

The authors received no financial support for the research and/or authorship of this article.

\section{REFERENCES}

1. Steuri R, Sattelmayer M, Elsig S, Kolly C, Tal A, Taeymans $\mathrm{J}$, et al. Effectiveness of conservative interventions including exercise, manual therapy and medical management in adults with shoulder impingement: a systematic review and meta-analysis of RCTs. Br J Sports Med 2017;51:1340-7.

2. Kuhn JE. Exercise in the treatment of rotator cuff impingement: a systematic review and a synthesized evidence-based rehabilitation protocol. J Shoulder Elbow Surg 2009;18:138-60.

3. Hawk C, Minkalis AL, Khorsan R, Daniels CJ, Homack D, Gliedt JA, et al. Systematic Review of Nondrug, Nonsurgical Treatment of Shoulder Conditions. J Manipulative Physiol Ther 2017;40:293-319.

4. Michener LA, Walsworth MK, Doukas WC, Murphy KP. Reliability and diagnostic accuracy of 5 physical examination tests and combination of tests for subacromial impingement. Arch Phys Med Rehabil 2009;90:1898-903.

5. Institute for Work and Health. Disabilities of the Arm, Shoulder and Hand outcome measure. Available at: http:// www.dash.iwh.on.ca/. [Accessed: December 25, 2018].

6. Dutton M. Orthopedic examination, evaluation and intervention. 3rd ed. New York: McGraw Hill; 2004. 
7. Beaton DE, Katz JN, Fossel AH, Wright JG, Tarasuk V, Bombardier C. Measuring the whole or the parts? Validity, reliability, and responsiveness of the Disabilities of the Arm, Shoulder and Hand outcome measure in different regions of the upper extremity. J Hand Ther 2001;14:128-46.

8. Düger T, Yakut E, Öksüz Ç, Yörükan S, Semin Bilgütay B , Ayhan Ç, et al. Kol,Omuz ve El sorunları (Disabilities of the Arm,Shoulder and Hand - DASH) Anketi Türkçe uyarlamasının güvenirliği ve geçerliği. Fizyoterapi Rehabilitasyon 2006;17:99-107.

9. Tate AR, McClure PW, Young IA, Salvatori R, Michener LA. Comprehensive impairment-based exercise and manual therapy intervention for patients with subacromial impingement syndrome: a case series. J Orthop Sports Phys Ther 2010;40:474-93.

10. Michener LA, Snyder Valier AR, McClure PW. Defining substantial clinical benefit for patient-rated outcome tools for shoulder impingement syndrome. Arch Phys Med Rehabil 2013;94:725-30.

11. Michener LA, McClure PW, Sennett BJ. American Shoulder and Elbow Surgeons Standardized Shoulder Assessment Form, patient self-report section: reliability, validity, and responsiveness. J Shoulder Elbow Surg 2002;11:587-94.

12. Celik D, Atalar AC, Demirhan M, Dirican A. Translation, cultural adaptation, validity and reliability of the Turkish ASES questionnaire. Knee Surg Sports Traumatol Arthrosc 2013;21:2184-9.

13. Ware JE Jr. SF-36 health survey update. Spine 2000;25:3130-9.

14. Bang MD, Deyle GD. Comparison of supervised exercise with and without manual physical therapy for patients with shoulder impingement syndrome. J Orthop Sports Phys Ther 2000;30:126-37.

15. Camargo PR, Alburquerque-Sendín F, Avila MA, Haik MN, Vieira A, Salvini TF. Effects of Stretching and Strengthening Exercises, With and Without Manual Therapy, on Scapular Kinematics, Function, and Pain in Individuals With Shoulder Impingement: A Randomized Controlled Trial. J Orthop Sports Phys Ther 2015;45:984-97.
16. Kachingwe AF, Phillips B, Sletten E, Plunkett SW. Comparison of manual therapy techniques with therapeutic exercise in the treatment of shoulder impingement: a randomized controlled pilot clinical trial. J Man Manip Ther 2008;16:238-47.

17. Guimarães JF, Salvini TF, Siqueira AL Jr, Ribeiro IL, Camargo PR, Alburquerque-Sendín F. Immediate Effects of Mobilization With Movement vs Sham Technique on Range of Motion, Strength, and Function in Patients With Shoulder Impingement Syndrome: Randomized Clinical Trial. J Manipulative Physiol Ther 2016;39:605-15.

18. Delgado-Gil JA, Prado-Robles E, Rodrigues-de-Souza DP, Cleland JA, Fernández-de-las-Peñas C, AlburquerqueSendín F. Effects of mobilization with movement on pain and range of motion in patients with unilateral shoulder impingement syndrome: a randomized controlled trial. J Manipulative Physiol Ther 2015;38:245-52.

19. Conroy DE, Hayes KW. The effect of joint mobilization as a component of comprehensive treatment for primary shoulder impingement syndrome. J Orthop Sports Phys Ther 1998;28:3-14.

20. Mulligan EP, Huang M, Dickson T, Khazzam M. The effect of axioscapular and rotator cuff exercise training sequence in patients with subacromial impingement syndrome: A randomized crossover trial. Int J Sports Phys Ther 2016;11:94-107.

21. Shire AR, Stæhr TAB, Overby JB, Bastholm Dahl M, Sandell Jacobsen J, Høyrup Christiansen D. Specific or general exercise strategy for subacromial impingement syndromedoes it matter? A systematic literature review and meta analysis. BMC Musculoskelet Disord 2017;18:158.

22. Bdaiwi AH, Mackenzie TA, Herrington L, Horsley I, Cools AM. Acromiohumeral Distance During Neuromuscular Electrical Stimulation of the Lower Trapezius and Serratus Anterior Muscles in Healthy Participants. J Athl Train 2015;50:713-8.

23. Smith MV, Calfee RP, Baumgarten KM, Brophy RH, Wright RW. Upper extremity-specific measures of disability and outcomes in orthopaedic surgery. J Bone Joint Surg [Am] 2012;94:277-85. 